\title{
LA JUSTIFICACIÓN DIALÉCTICA DE LA REFUTACIÓN AL ELEATISMO EN FÍSICA I, 2-3
}

\author{
Carlos Carrasco Meza \\ Universidad Andrés Bello \\ cgcarrasco@uc.cl
}

\begin{abstract}
RESUMEN / ABSTRACT
Este trabajo tiene por objetivo mostrar el carácter dialéctico de la refutación al eleatismo en Física I, 2-3. En primer lugar, se argumenta que la dialéctica aristotélica puede llegar a constituir un método de investigación científica, ya que permite esclarecer los principios de una ciencia particular. En segundo lugar, se muestra que la refutación al eleatismo en Física I 2-3 solo se justifica en el marco de una argumentación dialéctica, ya que esta refutación constituye un método de esclarecimiento de los principios de la física.

Palabras clave: dialéctica, método, refutación, eleatismo, Física I, 2-3.

\section{DialeCtical JustifiCATION OF ELEATISM's REFUTATION IN PHYSICS I, 2-3}

This paper aims at expounding the dialectical character of Eleatism's refutation in Physics I 2-3. First, it will be argued that the Aristotelian dialectic can constitute a method of scientific investigation. Secondly, it will be shown that the refutation of Eleatism in Physics I 2-3 can be justified onlywithin the framework of a dialectical argumentation, since this refutation constitutes a method of clarification of physical principles.
\end{abstract}

KEYWORDS: dialectic, method, refutation, Eleatism, Physics I, 2-3.

\section{La dialéctica como método de investigación científica}

\subsection{Conocimiento de los principios}

RT

Aristóteles desarrolla en Analíticos Posteriores un modelo de ciencia, de acuerdo al cual una ciencia particular es un conjunto de procesos deductivos que se sustentan en la verdad de ciertos principios propios de esa ciencia. Estos principios propios son de dos tipos: las hipótesis (vं género de objetos a investigar, y las definiciones (ópı $\sigma \mu o i ́)$ de los objetos que pertenecen a ese género ${ }^{1}$. La definición debe ser causal, i.e., debe definir el objeto mediante ciertas 
causas, pues es el conocimiento de las causas lo que constituye conocimiento científico ${ }^{2}$. Sin embargo, el tipo de objeto de cada ciencia especifica el tipo de definición que ésta requiere: A diferencia de las definiciones matemáticas, que solo deben incluir la causa formal, las definiciones físicas deben incluir las cuatro causas: material, formal, eficiente y final ${ }^{3}$. Esto implica que el establecimiento de las definiciones físicas requiere mayor trabajo de investigación que el establecimiento de las definiciones matemáticas. En lo que respecta a las hipótesis de la ciencia física, Aristóteles considera empíricamente evidente la existencia de la naturaleza y los entes naturales ${ }^{4}$.

A partir del establecimiento de la existencia y definición de los objetos de la ciencia, se procede a la deducción de las propiedades esenciales contenidas en la misma definición. La deducción procede respetando y guiándose por los principios comunes a todas las ciencias, a saber: el principio de no contradicción y el del tercero excluido 5 . El silogismo científico, tematizado en Analíticos Primeros, es el proceso deductivo mediante el cual las propiedades esenciales de un objeto científico son reveladas a partir de los principios propios de esa ciencia. Ahora bien, la deducción corresponde solo a una fase de la investigación científica, que, como tal, supone el establecimiento previo de los principios propios desde los cuales procederá la demostración ${ }^{6}$. De este modo, surge la pregunta por la manera en que ambas fases de la investigación se relacionan, a saber, la fase constituyente de los principios y la fase demostrativa de las propiedades contenidas en los principios ya constituidos. La respuesta a esta interrogante atañe directamente a uno de los problemas centrales de la epistemología aristotélica, que se puede formular de la siguiente manera: ¿cómo probar la verdad de los principios sin recurrir a la demostración, la cual no puede comenzar antes de que se establezcan los principios que la sustentan? La mayor dificultad epistemológica de un modelo de ciencia fundacionalista como el aristotélico es que, por una parte, el conocimiento de los principios debe sustentarse en un saber previo $^{7}, \mathrm{y}$, por otra parte, la verdad de los principios propios de una ciencia particular no puede ser deducida, ya que toda deducción debe partir precisamente de principios ya establecidos.

Aristóteles formula el problema como trilema: 1) Si el establecimiento de los principios de la ciencia solo puede ser realizado recurriendo a principios previos, caemos en un regreso al infinito que no constituye conocimiento de ningún tipo. Aristóteles señala claramente en Metafísica II 2 que las causas -formal, material, eficiente y

Analiticos Posteriores II, 10, 11; I 2, 71b10, 25-30; Física I, 1, 184a10; Metafísica I, 1. Física II 2, 7. Metafisica VI, 1, 1025b30-1026a5.

Física, I, 2, 185a12-13; II, 1, 193a3-9; VIII, 3. 253a32-253b6; 254a23-30.

Metafisica IV, 3.

Berti, E. Las razones de Aristóteles. Trad. Horacio A. Gianneschi y Maimiliano Monteverdi. Buenos Aires: Oinos, 2008, p. 29. Barnes, J. “Aristotle's Theory of Demonstration”, pp. 81-2. Wieland, W. Die aristotelische Physik. Göttingen: Vandenhoeck \& Ruprecht, 1992, p. 53.

7 Analíticos Posteriores, I,1. Ver Cassini, A. "El fundacionismo de la epistemología aristotélica". Crítica, Revista Hispanoamericana de Filosofia, vol. XX, n58 (abril 1988), pp. 67-95. 
final- de un objeto no pueden ser infinitas, ni en serie ni en especies (994a1-2). Esto concierne directamente al conocimiento de los objetos físicos, pues es precisamente la definición de éstos la que incluye las cuatro causas. 2) Si, por otra parte, los principios son establecidos mediante una demostración circular, que los deduce desde las consecuencias del mismo sistema, caemos en la imposibilidad de diferenciar el principio de aquello que éste explica, de modo que, si A implica B y B implica $\mathrm{C}$, y suponemos que $\mathrm{C}$ implica $\mathrm{A}$, entonces $\mathrm{C}$ implicará $\mathrm{B}$, pues $\mathrm{A}$ implica $\mathrm{B}$. Y ya que B implica $\mathrm{C}$, llegamos a que $\mathrm{C}$ implica $\mathrm{C}$, lo que es una mera tautología que no constituye conocimiento científico. 3) La última posibilidad es que el establecimiento de los principios sea convencional, es decir, que los principios funden lo que hoy denominamos un 'sistema hipotéticodeductivo'. Esta última posibilidad es inadmisible, ya que los principios, si han de valer como tales, deben ser verdaderos por sí $\operatorname{mismos}^{8}$. Así formulado, el trilema constituye un argumento escéptico que pretende refutar la posibilidad del fundacionalismo9.

Aristóteles resuelve el trilema admitiendo un principio heurístico que-solo siendo extremadamente cautos- podemos considerar una variante de la tercera posibilidad: los principios son establecidos, pero no hipotéticamente, ni convencionalmente, ni menos arbitrariamente, sino por un proceso complejo que involucra el análisis de la experiencia y del lenguaje. En los Analíticos Posteriores (I, 2, 71b29-72b5; II, 19) se establece la

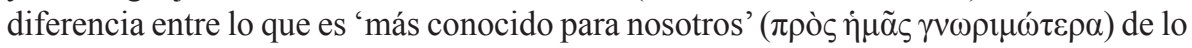
que es 'más conocido sin más' ( $\dot{\alpha} \lambda \lambda \tilde{\omega} \varsigma \gamma \nu \omega \rho \mu \omega ́ \tau \varepsilon \rho \alpha)$, relacionando lo más conocido para nosotros con el conocimiento empírico de lo singular y lo más conocido sin más -o 'por naturaleza'- con el conocimiento intelectual de lo universal. En concordancia con esto, en Analíticos Posteriores II, 19 y Física I, 1 (184a16-154b15), Aristóteles remarca tanto el carácter analítico como sintético que implica el conocimiento, que procede separando lo que en la experiencia se muestra como indiferenciado, descubriendo, de este modo, las conexiones causales entre las cosas y los principios que expliquen esas cosas, y el consiguiente establecimiento de la definición universal ${ }^{10}$. Ahora bien, la verdad de los principios es aprehendida solo por la inteligencia (voũ $\zeta$ ), que los capta en un acto único de intelección ${ }^{11}$. Sin embargo, la captación intelectiva de los principios será tanto más probable cuanto más despejado esté el camino que va desde los principios hacia las cosas que éstos explican. Esto supone un arduo proceso previo de investigación, que consiste fundamentalmente en un análisis de la experiencia sensible y del lenguaje, pues éstos constituyen precisamente aquello que los principios deben explicar, es decir, son el único terreno desde el cual es posible rastrear las conexiones causales. El análisis de la experiencia y del lenguaje forman parte de un mismo proceso investigativo que,

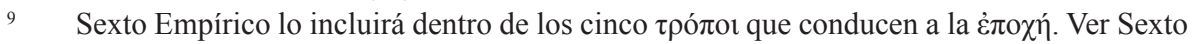
Empírico, Esbozos Pirrónicos, I, XV.

10 Una discusión en profundidad sobre estos pasajes se encuentra en Bolton, R. "Aristotle's Method in Natural Science: Physics I", en Lindsay Judson. Aristotle's Physics. A Collection of Essays. Oxford Clarendon Press, 2003, pp. 1-29.

$11 \quad$ Metafisica IX, 10. Berti (2008), pp. 30-36. 
si bien no garantiza la realización de la captación intelectiva de los principios, sí la vuelve más probable.

En lo que respecta al análisis de la experiencia sensible, Aristóteles considera que la experiencia repetida de los singulares, conservada luego en la memoria y actualizable en cada momento por la imaginación, favorece la decantación del universal en el alma $^{12}$. Ahora bien, la decantación del universal puede ser, y tiene que ser, favorecida por el esclarecimiento de los conceptos mediante los cuales denominamos en cada caso aquello que se muestra en la experiencia, y por el análisis crítico de las opiniones acerca de aquello que se da en la experiencia. En efecto, todo ser racional dispone de cierto conocimiento precientífico que se refleja en cierta configuración del lenguaje. La célebre tesis de Wieland respecto a este problema, es que el conocimiento de los principios consiste fundamentalmente en la diferenciación de un presaber indiferenciado ${ }^{13}$. Este preconocimiento es aquello que Aristóteles caracteriza como 'más conocido para nosotros' y 'confuso', y cuyo análisis y depuración conducen a una diferenciación que revela lo que es 'más conocido sin más'.

No pretendemos aquí dar una respuesta definitiva a este célebre problema, sino solo destacar que el camino hacia los principios implica no solo una teoría del conocimiento empírico, que permita discriminar y poner orden en la evidencia sensible, sino también una teoría de la argumentación, que permita analizar y evaluar aquellas verdades o preverdades contenidas en el lenguaje. En lo que respecta al análisis lingüístico como método de investigación científica, Aristóteles expone en los Tópicos una teoría según la cual la dialéctica puede ser entendida, en cierto sentido, como método argumentativo que coadyuva a conocer los principios de cualquier ciencia. Esto último exige una explicación más detallada, que intentaremos resumir a continuación.

\subsection{La dialéctica como método de investigación de los principios}

Aristóteles comienza los Tópicos caracterizando la dialéctica, de manera general, como un método $(\mu \varepsilon \dot{\theta} \theta \delta \delta \varsigma)$ que permite argumentar sobre cualquier problema propuesto. Se trata de un método de argumentación que faculta para discutir, lo que presupone siempre un interlocutor que defiende la posición contraria a la de uno, y un contexto de discusión. Al igual que la apodíctica, la dialéctica procede por deducción, solo que el punto de partida de ella no son premisas verdaderas por sí mismas, sino más bien premisas cuya verdad es relativa a la opinión de cierto grupo de individuos. En efecto, Aristóteles inicia los Tópicos diferenciando el silogismo dialéctico del silogismo científico, es decir, de la demostración, señalando que este último parte de premisas

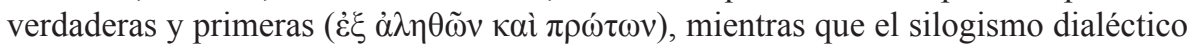

12 Para un análisis detallado de este proceso me permito remitir a Siggen, M. La Méthode Expérimentale selon Aristote. Les Presses universitaires, 2006.

13 Wieland, pp. 59-85. 


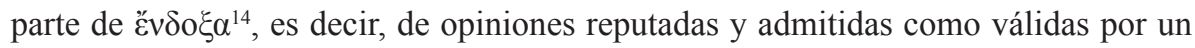
grupo de personas. No se trata de que la argumentación dialéctica consista en defender o atacar las opiniones de ese grupo, sino más bien de que los $̌ ้ v \delta o \xi \alpha$ constituyen el marco de referencia incuestionable en el que se enmarca la discusión dialéctica.

Respetando un marco de opiniones comunes, la argumentación dialéctica inicia desde una oposición de proposiciones respecto a una misma cosa, es decir, desde el establecimiento de un problema $(\pi \rho{ } \beta \lambda \eta \mu \alpha)^{15}$. Un problema es una pregunta de tipo alternativo, es decir, que interroga presentando dos alternativas mutuamente excluyentes, e.g., ¿animal terrestre bípedo es definición de hombre, o no? El problema puede presentar alternativas contrarias o mutuamente contradictorias, siendo este último caso aquél que Aristóteles remarca como más propio de la argumentación dialéctica. En efecto, en Analíticos Posteriores (I, 2, 72a8-14) leemos que la demostración científica puede partir de un enunciado afirmativo o de uno negativo, mientras que la discusión dialéctica parte de la consideración tanto del enunciado afirmativo como del correspondiente enunciado negativo. Es decir, la argumentación dialéctica inicia con el establecimiento de un problema que plantea interrogativamente una oposición de

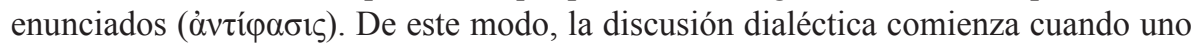
de los interlocutores propone un enunciado -que no cuestiona el marco de referencia dado por los $\check{\varepsilon} v \delta o \xi \alpha$, aceptados por ambos interlocutores y el auditorio que presencia la discusión- y el otro interlocutor propone el enunciado opuesto. Ambos argumentan intentando que el oponente incurra en contradicción, la cual puede ser básicamente de tres tipos: 1) respecto a las premisas de las que parte el interlocutor, es decir, una contradicción entre sus premisas y las conclusiones derivadas de sus premisas, 2) respecto a afirmaciones previas del interlocutor, es decir, una contradicción entre algo previamente afirmado y algo afirmado posteriormente y 3) una contradicción entre las

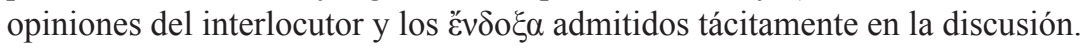

En Tópicos I, 2 Aristóteles atribuye tres usos a la dialéctica: "para ejercitarse

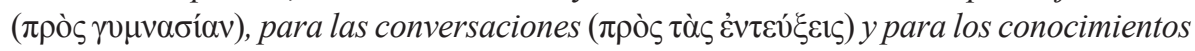

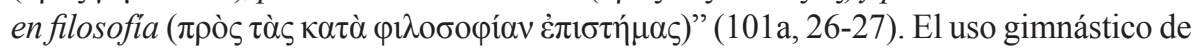
la dialéctica es el que Parménides recomienda a Sócrates y lleva a cabo en la segunda parte del diálogo platónico homónimo: "esfuérzate y ejercitate ( $\sigma \alpha v \tau o ̀ v ~ \gamma u ́ \mu v \alpha \sigma \alpha$ ) más, a través de esa práctica aparentemente inútil" (Parménides, 135d), es la recomendación de Parménides a Sócrates, si éste pretende habérselas mejor con la realidad inteligible. Aristóteles recoge de este diálogo la noción tradicional de la dialéctica, entendida como ejercicio argumentativo que puede ser practicado individualmente y que prepara para la posterior discusión real en los encuentros forenses y deliberativos. A diferencia de Sócrates y Parménides (personaje del diálogo), Aristóteles se alinea con Zenón, atribuyendo a la dialéctica la capacidad de argumentar no solo sobre entidades inteligibles, 
sino sobre cualquier entidad ${ }^{16}$. Como veremos, la formalidad de la dialéctica da la posibilidad de aplicarla al estudio de toda entidad, incluso a la realidad sensible. Pero Aristóteles adopta la recomendación del Parménides platónico, de que la dialéctica debe proceder deduciendo no solo desde un único enunciado, como lo hacía Zenón, sino también desde el enunciado opuesto. El estudiante de dialéctica deberá aprender a desarrollar las consecuencias que se derivan de adoptar las dos posiciones enfrentadas en cada problema. Esta capacidad de la dialéctica mostrará su utilidad tanto en la práctica real de la argumentación forense y deliberativa, como en la utilidad filosófica que Aristóteles le atribuye.

El segundo uso de la dialéctica es el de la práctica dialéctica, en la que el estudiante sale al encuentro ( $(\varepsilon \tau \tau \varepsilon v \xi ı)$ ) de un contrincante, planteando el enunciado contrario al del interlocutor e intentando hacer que caiga en contradicción. Para lograrlo, el estudiante tendrá que discutir respetando los $\varepsilon^{2} v \delta o \xi \alpha$ que admiten él mismo, el interlocutor y el auditorio. Aristóteles recomienda inventariar ( $\kappa \alpha \tau \alpha \rho \theta \mu \varepsilon \tilde{v} v)$ y confeccionar listas

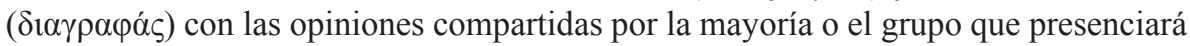
la discusión, clasificándolas bajo ciertos epígrafes y consignando también las opiniones individuales de ciertos sabios respecto al tema en discusión ${ }^{17}$. Junto con el estudio de los

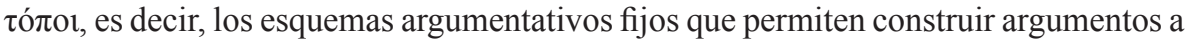
partir de dichas opiniones clasificadas, el estudiante será capaz de argumentar contra el interlocutor, mostrando que éste cae en contradicción con los $\varepsilon$ $v \delta o \xi \alpha$, con sus premisas particulares, o con ciertas afirmaciones previas. Se trata de poner en práctica el método aprendido en la ejercitación dialéctica, solo que ahora cada interlocutor adopta una única posición.

Pero Aristóteles reconoce también en la dialéctica un uso filosófico, que no es sino una aplicación particular de la misma técnica argumentativa aprendida por el estudiante en la ejercitación y puesta en práctica en los encuentros. La diferencia respecto a los otros usos es que éste es capaz de constituir conocimiento científico:

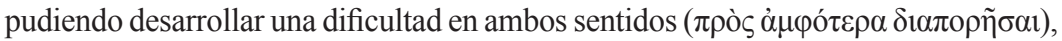
discerniremos más fácilmente lo verdadero y lo falso en cada cosa. Pero es que además es útil para las cuestiones primordiales propias de cada conocimiento. En efecto, a partir de lo exclusivo de los principios internos al conocimiento en cuestión, es imposible decir nada sobre ellos mismos, puesto que los principios son primeros con respecto a todas las cosas, y por ello es necesario discurrir $(\delta 1 \varepsilon \lambda \theta \varepsilon i \tau v)$ en torno a ellos a través de las cosas plausibles concernientes a cada uno de ellos. Ahora bien, esto es propio o exclusivo de la dialéctica: en efecto, al ser adecuada para examinar <cualquier cosa $>$, abre el camino a los principios de todos los métodos (Tópicos I, 2, 101a35-101b5). 
En primer lugar, la dialéctica es útil para la filosofía porque enseña a desarrollar las aporías en ambas direcciones y, de este modo, revelar lo verdadero y lo falso. Se trata de deducir las consecuencias de la admisión de una proposición filosófica y las de su negación, para verificar o falsear estas proposiciones. En efecto, al extraer las consecuencias de una proposición, se puede llegar a una contradicción entre las consecuencias y esa proposición, entre las consecuencias y otras admisiones previas, o entre las consecuencias y los $̌$ है $v \delta o \xi \alpha$ que arbitran la argumentación dialéctica. En cualquiera de estos casos la contradicción es señal de falsedad, lo que obliga a cambiar la proposición original o a modificar ciertas admisiones. Este mismo proceso se puede llevar a cabo desde la proposición antagónica. De este modo, el método diaporético es útil para la filosofía debido a su carácter peirástico, es decir, examinador de una proposición y su contraria, ya que permite revelar cuán fiable es la proposición defendida, en caso de que no se deje contradecir fácilmente, y cuán débil es la proposición a refutar, en caso de que sea refutada fácilmente.

Por lo general, Aristóteles no busca refutar completamente una proposición filosófica, sino más bien mostrar su parcial aproximación a la verdad. Esto se consigue al mostrar que sus interlocutores filosóficos, que suelen ser filósofos connotados como Platón y los presocráticos, han usado una terminología imprecisa, de modo que el examen de sus afirmaciones exige un análisis semántico de los términos que usan. Este análisis puede colaborar en la refutación definitiva de una proposición, precisamente mostrando que el uso de los conceptos por parte del filósofo no es consistente. Pero el análisis semántico también permite evaluar posibles maneras de salvar las afirmaciones de un filósofo, revelando posibles interpretaciones alternativas. De este modo, Aristóteles suele concluir que sus predecesores filosóficos se han expresado de manera obscura, pero que han atisbado en cierto grado la verdad ${ }^{18}$.

Ahora bien, cuando la argumentación dialéctica parte de la oposición entre una proposición y su contradictoria -y no solo contraria-, este método de argumentación permite adquirir conocimiento, ya que desarrollando uno de los lados de la cuestión y mostrando su falsedad, queda demostrada refutativamente la verdad de la proposición contradictoria. El rol cognoscitivo de este tipo de dialéctica lleva a autores como Berti a hablar de una 'dialéctica fuerte', en oposición a una 'dialéctica débil', cuyo representante habría sido, de acuerdo a Aristóteles, Sócrates:

Aquél [Sócrates], sin embargo, pretendía con razón encontrar el qué-es, pues pretendía razonar por silogismos y el qué-es constituye el punto de partida de los silogismos. Pues la dialéctica no era entonces lo suficientemente vigorosa como para ser capaz de investigar los contrarios aparte del qué-es, y si la misma ciencia se ocupa de los contrarios (Metafisica XIII, 4, 1078b24-27).

Berti se apoya en este pasaje para afirmar que la debilidad de la dialéctica socrática consistía en su imposibilidad de discurrir prescindiendo de la esencia, es decir, en su 
necesidad de restringirse al principio verdadero y solo desde él extraer las consecuencias. Paradójicamente, la deficiencia de la dialéctica socrática radicaba en su naturaleza apodíctica. Heredando la innovación de Platón (encarnado en el Parménides del diálogo), Aristóteles desarrolla una dialéctica que permite discurrir desde cualquier premisa. Esta neutralidad y universalidad es una condición necesaria para que la dialéctica se constituya como método de argumentación científica, pues solo careciendo ella misma de doctrina, es decir, ignorando la verdad de cualquier principio, podrá ser aplicada en la demostración y refutación de cualquier principio.

La segunda razón por la cual la dialéctica aristotélica puede constituir un método de investigación científica es una consecuencia necesaria de su carácter diaporético; la dialéctica es útil para las ciencias, porque "abre el camino a los principios". La posibilidad de la dialéctica de favorecer la mostración de la verdad de los principios estriba en su función depuradora, es decir, en la posibilidad que ella ofrece de verificar ciertas tesis y falsear otras, estableciendo, de este modo, la verdad relativa de ciertas suposiciones y la falsedad de otras. Por este método, la dialéctica permite discernir lo verdadero y lo falso, limpiando el camino que conduce hacia los principios de todo rastro de falsedad y destacando todo rastro de verdad. De este modo, la dialéctica coopera con la inteligencia en el establecimiento de los principios propios de una ciencia, la primera haciendo más probable la revelación de la verdad de los principios, la segunda aprehendiendo la verdad de los principios.

En resumen, la dialéctica 'fuerte' es capaz de cumplir un rol científico, consistente en favorecer la intuición de los principios por la inteligencia, y lo hace en base a tres procedimientos lógicos: el análisis semántico, la diaporética y la reducción a contradicción. A continuación, examinaremos la aplicación de la dialéctica fuerte en la argumentación de Física I, 2-3, que tiene por finalidad refutar la tesis eleata de la unidad del ser y, de este modo, demostrar la verdad de la proposición contradictoria correspondiente, a saber: que el ser se dice de muchas maneras.

\section{Argumentación dialéctica contra el eleatismo en Física I, 2-3}

En esta sección intentaremos ejemplificar el uso de la dialéctica por parte de Aristóteles, analizando su argumentación contra el eleatismo en Física I, 2-3. En la sección anterior nos hemos referido a los $\varepsilon^{\prime} v \delta o \xi \alpha$ como opiniones incuestionables que ofrecen el marco de referencia para la argumentación dialéctica. Ahora bien, dado que la tesis eleata es caracterizada por Aristóteles como opuesta a todas las opiniones admitidas, es decir, a todos los $\check{c} v \delta o \xi \alpha$, afirmar que la argumentación contra el eleatismo es dialéctica parece estar en contradicción con nuestra descripción del método dialéctico en la sección anterior. Debemos retomar, por tanto, aquella exposición y profundizar en la noción

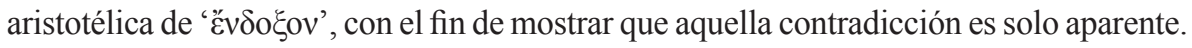


Berti propone entender ' $\check{v} v \delta o \xi o v$ ' como una opinión que es connotada o afamada, y solo en sentido derivado es verosímil o probable ${ }^{19}$. Christof Rapp y Tim Wagner proponen diferenciar diversas clases de $\varepsilon^{\prime} \delta \delta$ o $\xi$, dependiendo de qué tipo o clase de grupo es el que considera admisible (anerkannt) la proposición en cuestión ${ }^{20}$. Robin Smith comparte esta lectura de los $\check{\varepsilon} v \delta o \xi \alpha$, entendidos como premisas aceptables (acceptable) por cierto grupo, es decir, cuya admisibilidad tiene un carácter relativo ${ }^{21}$. Esto origina la dificultad de que una proposición filosófica, es decir, defendida por ciertos especialistas, podría ser admitida solo por algunos sabios, la mayoría, o todos, pero no por la mayoría no-sabia. Sin embargo, Aristóteles relaciona ambos criterios de clasificación de los ع̌ $v \delta o \xi \alpha$ señalando que, en el caso de una proposición defendida por ciertos sabios, esta proposición será dialéctica solo si pudiese ser eventualmente admitida por la mayoría no-sabia, es decir, si la proposición no es paradójica $(\pi \alpha \rho \alpha ́ \delta o \xi o \varsigma)^{22}$. Se trata de un criterio de demarcación que sigue basándose en la admisión relativa a un grupo, solo que en este caso la admisión es potencial. Así como nadie comenzaría una discusión dialéctica negando lo que es manifiesto para todos, nadie comenzaría tampoco afirmando lo que es absurdo para todos. En efecto, si bien la mayoría no tiene conocimientos de medicina, no cuestiona al médico, sino que acata lo que dice; aquello porque es sabio (en comparación al saber de la mayoría), esto porque no dice nada paradójico. La duda respecto a la admisibilidad de una proposición, es decir, donde se destruye la posibilidad de iniciar una argumentación dialéctica tomando esa proposición como है $v \delta o \xi o v$, es en los extremos: cuando la proposición niega lo que todos aceptan o cuando la proposición afirma lo que todos niegan.

Esto no quiere decir que pueda haber argumentación dialéctica solo cuando hay perfecta correspondencia entre las opiniones de la mayoría y las opiniones de los sabios, sino solo que la proposición dialéctica no debe ser contraintuitiva, es decir, no debe ir en contra del sentido común. Rapp y Wagner ponen como ejemplo de afirmación contraintuitiva la negación del movimiento y la pluralidad por parte de los eleatas, o la negación de la debilidad de la voluntad por parte de Sócrates ${ }^{23}$. En efecto, en varios

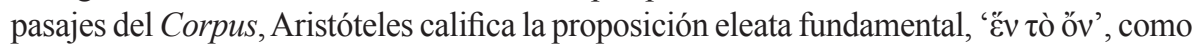

19 Berti (2008), pp. 41-43.

$20 \quad$ Ver Rapp, Ch.; Wagner, T. Aristoteles, Topik. Stuttgart: Reclam, 2004, pp. 21-2: “'Ser-admisible' es una expresión relativa, de modo que, tomada en serio, siempre se tiene que especificar

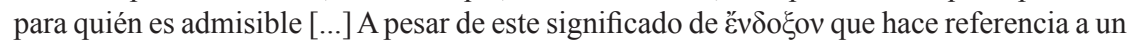

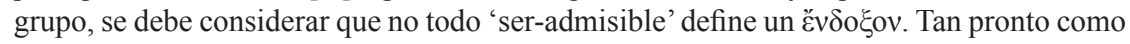
se abandona el ámbito de las opiniones generales o claramente mayoritarias, una opinión cuenta como admisible solo si ella es admitida por todos o la mayoría de especialistas, o, al menos, por los más connotados. Pero esto tampoco es suficiente: opiniones expuestas de manera particular valen como admisibles solo cuando no contradicen la opinión de la mayoría o cuando no hay en la mayoría una opinión establecida respecto al tema en cuestión" (trad. propia).

21 Smith, R. Aristotle. Topics. Books I and VIII. Oxford: Clarendon Press, 1997, pp. xxiii-xxiv.

22 Tópicos I, 10, 104a8-11.

23 Rapp; Wagner, p. 22. 
'erística' y la considera contraintuitiva ${ }^{24}$, lo que implica que no puede ser considerada un $\varepsilon \vee \delta$ o ov que sirva de punto de referencia para una discusión acerca de la naturaleza.

La discrepancia que da origen al problema dialéctico no se funda en la afirmación de algo paradójico o contraintuitivo, sino en la diferencia de dos opiniones basadas ambas en el sentido común. Puede ocurrir, sin embargo, que alguien emita un juicio que no solo vaya en contra de una opinión particular, sino que sea paradójico por ser contrario a todas las opiniones compartidas por la mayoría. En tal caso, probablemente nadie prestará asentimiento a tal afirmación, pero el caso es otro cuando su defensor es alguien conocido en el terreno de la filosofía. En Tópicos I, 11, Aristóteles denomina 'tesis' ( $\theta \varepsilon \dot{\sigma} \sigma \varsigma)$ a un juicio paradójico ( de renombre, y pone como ejemplos la tesis de Antístenes de que no es posible el contradecir, la tesis de Heráclito de que todo está en movimiento, y la tesis de Meliso

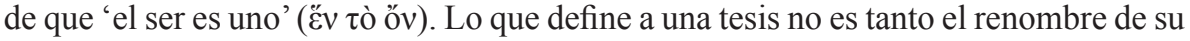
defensor, sino más bien el carácter paradójico de la proposición respecto a la opinión

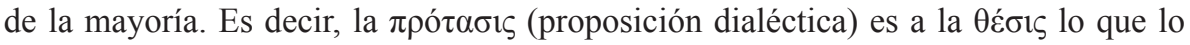

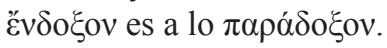

Habiendo hecho estas aclaraciones, detallaremos a continuación los elementos presentes en la argumentación aristotélica contra el eleatismo, en Física I, 2-3, que nos permiten afirmar que se trata de una argumentación dialéctica.

\subsection{Carácter dialéctico de la refutación al eleatismo}

La Física de Aristóteles no es un tratado de filosofía sistemático, sino un conjunto de lecturas y apuntes de clases que, como tales, son un reflejo del work in progress del Aristóteles científico: Física I constituye una fundamentación dialéctica de la posibilidad de hacer una ciencia de la naturaleza ${ }^{25}$, mientras que Física II se ocupa de determinar los conceptos más generales -como los de ' $\varphi v ́ \sigma ı \varsigma '$, causa formal, material, eficiente y final-que servirán de base para la investigación posterior. La necesidad de establecer la posibilidad de llevar a cabo un estudio filosófico de la naturaleza, antes de comenzar la investigación propiamente tal, se explica por el contexto histórico-filosófico en que se inserta la reflexión aristotélica: Parménides y Meliso habían afirmado que el ser es uno e inmóvil, negando con ello no solo la posibilidad del estudio científico de lo natural, sino la existencia misma de lo natural. En efecto, para Aristóteles la naturaleza es el género de entidades cuyo principio de movimiento y reposo les es intrínseco ${ }^{26}$, de modo que negar la existencia de la pluralidad y el cambio implicaba negar la existencia

Física I, 2, 185a3-185a10. Ver también Física II, 1, 193a3-9; Física VIII, 3. 253a32-253b6; 254a23-30.

5 Ver Berti, E. "Les méthodes d'argumentation et de démostration dans la "Physique" (apories, phénomènes, principes)", en La Physique d'Aristote et les conditions d'une science de la nature, Paris: Librairie Philosophique J. Vrin, 1991, pp. 53-72.

Física I, 2, 185a12-14; II, 1, 192b13-15. 
de la naturaleza. El eleatismo se presenta, entonces, como la principal amenaza para el proyecto aristotélico. Esto explica la necesidad urgente de refutar definitivamente el eleatismo y que Aristóteles desarrolle esta refutación precisamente en Física I. Ahora bien, dado que la tesis de la inmovilidad la derivaron los eleatas de su ontología monista, Aristóteles concentra su atención en la refutación de la proposición monista de los eleatas, a saber: 'Ẽv $\tau$ ò őv'.

Como hemos mencionado antes, Aristóteles entiende la proposición eleata ' $\tilde{\varepsilon} v$

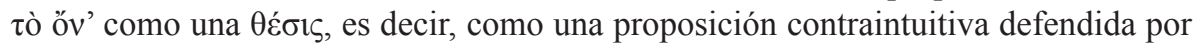
filósofos reputados. Dado que la tesis eleata contradice las opiniones de la mayoría respecto a cómo es la naturaleza, es decir, está en contradicción con ciertos čv $\delta$ o $\xi \alpha$, no puede ser considerada una proposición dialéctica; más aún, ella recusa toda posible discusión acerca del mundo natural. Sin embargo, la refutación aristotélica de la tesis eleata posee todas las características que hemos reconocido previamente en una argumentación dialéctica, y en el sentido 'fuerte' del término: 1) Aristóteles formula la

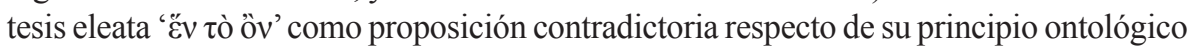

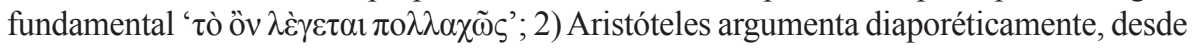
la verdad hipotética de la tesis eleata, para extraer consecuencias que contradigan esta tesis o ciertas afirmaciones de los eleatas; 3 ) el uso de la diaporética y la reducción a contradicción se sirve del análisis semántico de los términos empleados por los eleatas.

La argumentación contra la tesis eleata es una refutación, es decir, lo que Aristóteles

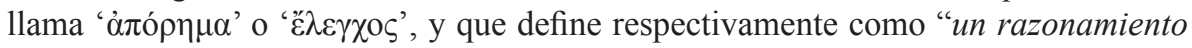
dialéctico en contradicción” (Tópicos VIII, 162a17-18) y "un razonamiento con contradicción en la conclusión" (Refutaciones Sofísticas 2, 165a2-3). El hecho de que Aristóteles considere la refutación como un tipo de argumento dialéctico implica que la refutación ha de tener las características formales de toda argumentación dialéctica. Se trata, entonces, de una aplicación particular de la dialéctica para probar la falsedad de una proposición, es decir, lo que en Metafísica IV llama "demostración refutatoria" $(\dot{\alpha} \pi \circ \delta \varepsilon \tilde{\xi} \xi \alpha \iota \dot{\varepsilon} \lambda \varepsilon \gamma \kappa \tau \iota \kappa \tilde{\omega} \varsigma)^{27}$. Esto indica, como veremos, que, al ser un tipo de demostración, la refutación es un método propio de la dialéctica fuerte. Por otra parte, en Física I, 2, 185a9-10 y I 3, 186a7-8 Aristóteles califica la argumentación de los eleatas como erística, ya que éstos parten de premisas falsas y las conclusiones que extraen no se siguen de sus premisas. Esto concuerda con la clasificación de los tipos de razonamiento y de argumentos en Tópicos y Ref. Sofísticas, de acuerdo a la cual los argumentos erísticos

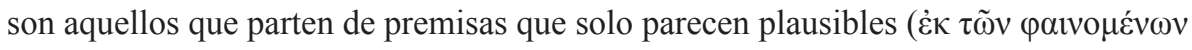
$\dot{\varepsilon} v \delta$ ó $\xi \omega v)$, pero no lo son, y a partir de ellas extraen conclusiones incorrectamente.

En consecuencia, podemos sostener que la argumentación aristotélica contra los eleatas es una refutación dialéctica contra una tesis erística, entendiendo 'refutación', 'tesis' y 'erística', en el sentido técnico que define Aristóteles. 


\subsection{Esquema dialéctico de la refutación al eleatismo en Física I, 2-3}

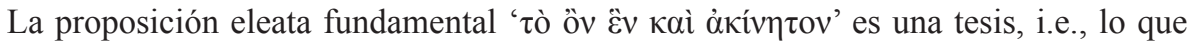
Aristóteles define como una proposición paradójica dicha por un filósofo de renombre. Su carácter paradójico se debe a la negación de la pluralidad y movilidad que contiene implícita en su afirmación de la unidad y la inmovilidad. Ya que la ciencia de la naturaleza ha de partir de la hipótesis de que el mundo natural existe, es decir, de que existe una pluralidad de cosas en movimiento, la tesis eleata niega la hipótesis fundamental de la ciencia de la naturaleza. Esto indica que la investigación de su verdad o falsedad no puede formar parte de la ciencia física propiamente constituida (Física I, 2, 184b25185a3). Su investigación tiene lugar solo en el proceso constituyente de la ciencia, es decir, durante la investigación de los principios de la ciencia natural, que es precisamente el tema central de Física $\mathrm{I}^{28}$.Física I 2-3 contiene la refutación del monismo eleata y Física I, 7-8 la refutación de la inmovilidad. En lo que sigue, nos concentraremos solo en la refutación del monismo eleata, pues la refutación de la inmovilidad no es sino una consecuencia de la anterior. Como veremos, la finalidad última del argumento contra el monismo eleata parece ser el constituir una demostración refutatoria de la verdad del $\pi \mathrm{o} \lambda \lambda \alpha \chi \tilde{\omega} \zeta$; en efecto, la tesis eleata monista, que se puede formular como 'el ser se dice de una única manera', es la proposición contradictoria del principio aristotélico 'el ser se dice de muchas maneras'. De este modo, el argumento aristotélico parece estar construido de tal forma, que la refutación del $\mu$ ov $\alpha \chi \tilde{\omega} \varsigma$ eleata implique la verdad del $\pi \mathrm{o} \lambda \lambda \alpha \chi \tilde{\omega} \varsigma$ aristotélico.

La refutación del monismo eleata se inserta en una argumentación dialéctica más amplia acerca de la cantidad y tipo de principios suficientes y necesarios para el estudio de los entes naturales. Siguiendo su método habitual de investigación, Aristóteles inicia los primeros capítulos de Física I con una discusión sobre los aportes hechos por sus predecesores en la aclaración de este problema particular. Desde el inicio se percibe el carácter dialéctico de la argumentación, pues Aristóteles formula estos aportes de modo tal, que la admisión de uno de ellos implique necesariamente el abandono de otro, es decir, como proposiciones mutuamente antagónicas:

Es necesario que haya o bien un único principio o bien múltiples, y que, si sólo hay uno, sea o bien inmóvil, como afirman Parménides y Meliso, o bien móvil, como sostienen los físicos [...] Pero, si hay múltiples principios, son o bien finitos o bien infinitos en número [...] De modo semejante plantean la cuestión los que investigan cuántos son los entes (őv $\tau \alpha$ ), ya que se ocupan de examinar si los principios primeros de los que ellos se constituyen son uno o múltiples, $\mathrm{y}$, si son múltiples, si finitos o infinitos. Así resulta que lo que se están planteando es si el principio y elemento es uno o múltiple (Física I, 2 184b15-25). 
Aristóteles interpreta el óv eleata como un tipo de $\alpha \rho \chi \eta^{29}$, lo que le permite insertar a los eleatas dentro de esta clasificación dicotómica y, a la vez, desarrollar un argumento general contra la noción eleata de principio: "si existe una única cosa y una en este sentido, no es ya un principio, pues principio es principio de alguna o algunas cosas" (Física I, 2, 185a3-10). Los eleatas caen en contradicción cuando afirman la unidad absoluta del $\dot{\alpha} \rho \chi \eta ́$, es decir, del őv, ya que un $X$ cualquiera solo puede ser considerado principio en la medida en que explica, es decir, en la medida en que hay una serie, cuya existencia se funda causalmente en la existencia de $X$ y que no se identifica plenamente con $X$. De este modo, los eleatas caen en una contradicción cuando afirman que hay principio y, a la vez, que no hay nada más que ese principio.

En el segundo argumento general contra el monismo de los eleatas, Aristóteles busca desarrollar las consecuencias que conlleva la admisión de la tesis eleata, con el fin de mostrar las contradicciones internas en que habrían incurrido al desarrollar su ontología. En este momento de la refutación acude Aristóteles al análisis semántico de los términos empleados por los eleatas al formular su doctrina:

el comienzo más apropiado de todos, dado que hay múltiples sentidos en que se dice que algo es, es preguntarse a cuál de los sentidos del 'es' se refieren los que afirman que todas las cosas son una (Física I, 2, 185a20-22).

La importancia de distinguir las diversas acepciones de un mismo término homónimo (ver Categorías 1), es explicada en Tópicos I, 15 y puesta en práctica de manera ejemplar en Metafísica V. Sin embargo, la doctrina del $\pi \mathrm{o} \lambda \lambda \alpha \chi \tilde{\omega} \varsigma$ y su tematización en la doctrina de las categorías, es aplicada de manera programática en muchos textos aristotélicos, siendo Física I, 2-3 un ejemplo de ello. Si los eleatas concibieron el 'őv' en cierto sentido, aquel sentido debe corresponder necesariamente a uno o algunos de los sentidos formalizados en la doctrina de las categorías. Aristóteles evalúa, entonces, tres posibilidades: Parménides y Meliso pudieron haber entendido el 'óv' como substancia, cantidad o cualidad. Ahora bien, en cualquiera de estos casos, la unidad del 'ôv' no puede ser absoluta, ya que todo entendimiento del 'ô $v$ ' supone la existencia de una substancia en la que inhiera y de la que se predique la cantidad o la cualidad, lo que implica admitir que el őv tiene más de un sentido ${ }^{30}$. Pero, de admitirlo, caerían en contradicción con su

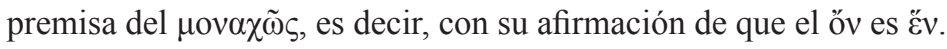

El tercer argumento general contra la tesis eleata es una continuación del argumento anterior. Mientras que aquél argumento estaba dirigido a mostrar los

29 La legitimidad de esta reducción se funda en la pluralidad de sentido de 'ả $\rho \chi \eta$ '. Ver Metafísica V, 1, donde Aristóteles muestra que la noción básica y general de 'principio’ es la de ser un 'desde-lo cual' algo está formado, surge, o puede ser explicado. Al menos el tercer sentido de 'principio' coincide con el tratamiento que los eleatas hicieron del öv.

30 Aristóteles no aborda aquí la posibilidad de interpretar el 'őv' eleata como una substancia pura, carente de determinaciones cuantitativas y cualitativas. Esta omisión se justifica porque Aristóteles aborda esta posibilidad en Física I, 3. 
problemas derivados de un entendimiento inadecuado del ǒv en su conexión con el ह̌v, la argumentación siguiente está dirigida a mostrar los problemas que se derivan del entendimiento eleata del हैंv en su conexión con el ôv:

Además, puesto que el uno mismo se dice de tantas maneras como el ser, hay que investigar en qué sentido dicen que todo es uno (Física I, 2, 185b5-7).

Aristóteles reconoce tres sentidos posibles para la unidad eleata: la unidad divisible, la unidad indivisible y la unidad de definición. Aristóteles pretende mostrar que la admisión de cualquiera de estos sentidos lleva a absurdos insalvables. Por ejemplo, si la unidad del todo es entendida como la unidad de una extensión continua, será divisible de manera indefinida, pues todo continuo es una pluralidad de partes potenciales. Pero si tiene partes, entonces admite diferencias. Esto implica que el ह̌v es $\pi 0 \lambda \lambda \alpha$, y ya que el őv es Ěv, el őv también es $\pi \mathrm{o} \lambda \lambda \alpha$. Pero esta conclusión está en contradicción con la

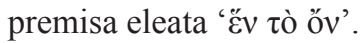

Tras esta crítica general dirigida contra ambos eleatas, Aristóteles comienza a diferenciar sus doctrinas (Física I, 3, 186a16-18). Esta diferenciación muestra que Meliso comprendió el ع̌v de manera material, y que Parménides lo entendió como unidad formal ${ }^{31}$. Sin embargo, Aristóteles dirige su atención principalmente a Parménides, pues considera la argumentación de Meliso 'grosera' y atribuye a Parménides mayor relevancia filosófica (Física I, 3, 186a9; Metafísica A, 5, 27-28). Como veremos, la importancia filosófica de Parménides estriba, en parte, en los efectos negativos de su filosofía: Parménides desarrolló una ontología restrictiva que negaba la posibilidad de fundar una filosofía natural. Es, por tanto, en la refutación específica del monismo parmenídeo donde se revela claramente el carácter dialéctico de la argumentación aristotélica, en el sentido más fuerte del término. En efecto, Aristóteles argumentará desde el principio metodológico que la refutación de la negación de su hipótesis fundamental, es una demostración de la verdad de esta última.

Aristóteles comienza la última parte de la refutación, intentando determinar en qué sentido pudo haber entendido Parménides el őv. Tras hacer esto, refuta la posibilidad de que el ǒv parmenídeo pueda ser entendido como atributo o como substancia sujeta a determinación. Habiendo refutado la posibilidad de entender el őv de Parménides en ambos sentidos, Aristóteles sostiene que la única posibilidad restante es suponer que Parménides haya entendido el ǒv en el sentido fundamental de 'existencia misma' (ő $\pi \varepsilon \rho$ őv), es decir, como 'aquello cuya esencia es el ser aquello que es' ${ }^{32}$, y el ह̌v como la unidad misma (ő $\pi \varepsilon \rho ~ \varepsilon ̋ v)$. Se trata del sentido más fundamental en el cual podría ser entendido el őv de Parménides y, con ello, su unidad. De este modo, la refutación exitosa

31 Lo que concuerda con la diferenciación de posturas en Metafisica A 5, 986b10-987a1, donde

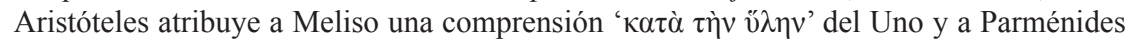

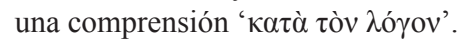

32 Ver Mansion, S. “Aristote, critique des Eléates”. Revue Philosophique de Louvain. Vol. 51, N³0 (1953) pp. 165-186, pp. 177-8. 
de esta última posibilidad parece conllevar la afirmación definitiva de la verdad de la proposición contradictoria, es decir, la verdad del $\pi \mathrm{o} \lambda \lambda \alpha \chi \tilde{\omega} \varsigma$.

Si el Ser parmenídeo es entendido como la existencia misma, se trata de una concepción puramente formal del Ser y su unidad. Ahora bien, si suponemos que el Ser parmenídeo ha de ser conocido, entonces ha de ser definible. Como todo concepto, la unidad formal del Ser de Parménides tendrá que ser analizable en las partes que integran su definición ${ }^{33}$. Aristóteles establece una analogía entre el Ser de Parménides y una unidad formal como 'hombre', la cual es analizable en sus partes definitorias 'animal' y 'bípedo' (Física I, 3, 186b14-18). Desde esta analogía, construye una reducción al absurdo que pretende mostrar que, aun entendido de esta manera, i.e., como ö $\pi \varepsilon \rho$ őv, el ő $v$ de Parménides puede ser dividido en partes conceptuales que no son meros accidentes ( $\sigma \nu \mu \beta \varepsilon \beta \eta \kappa o ́ \tau \alpha)$, sino que poseen la misma naturaleza esencial que el Ser. Es decir, Aristóteles busca probar que, si 'hombre' es analogable al ő $\pi \varepsilon \rho$ őv, y 'animal' y 'bípedo' son partes esenciales de 'hombre', entonces 'animal' y 'bípedo' representan análogamente cada una un ő $\pi \varepsilon \rho$ ő $\tau \imath$, Si se prueba esto, se habrá probado concomitantemente que estas partes, al ser cada una un ö $\pi \varepsilon \rho$ őv, será nuevamente divisible en sendos ő $\pi \varepsilon \rho$ ő $v \tau 1$.

Como toda reducción al absurdo, el argumento parte suponiendo la verdad de la proposición que se quiere rebatir, i.e., suponiendo que 'animal' y 'bípedo' no son cada uno un ő $\pi \varepsilon \rho$ őv, es decir, que son $\sigma v \mu \beta \varepsilon \beta \eta \kappa o ́ \tau \alpha$. Ahora bien, si suponemos que 'animal' y 'bípedo' son accidentes, podrán serlo o del ö $\pi \varepsilon \rho$ ǒv, i.e. de 'hombre', o de algún otro sujeto $X$. Aristóteles establece luego que 'accidente' significa aquello que puede pertenecer o no pertenecer a un sustrato, o aquello en cuya definición está contenida la definición del sujeto del que es accidente. El primer tipo de accidente es el separable, como por ejemplo, 'estar sentado' dicho de 'hombre'; en efecto, el accidente 'estar sentado' puede ser dicho de 'hombre' y otros sujetos. El segundo tipo es el accidente inseparable desde el punto de vista del predicado ${ }^{34}$, como 'ñata' es dicho de 'nariz' (Física I, 3, 186b18-23). En este caso no toda nariz es ñata, pero el accidente 'ñata' solo es predicable de una nariz. Tenemos, entonces, dos vías para desarrollar las consecuencias de la premisa, a saber: 1) 'animal' y 'bípedo' son accidentes de 'hombre' o 2) de otro sujeto $X$, a) 'animal' y 'bípedo' son accidentes separables o b) inseparables. La consideración de ambas vías origina cuatro combinaciones a evaluar en la reducción al absurdo: 1) a): 'animal' y 'bípedo' son accidentes separables de 'hombre'; 1) b) 'animal' y 'bípedo' son accidentes inseparables de 'hombre'; 2) a) 'animal' y 'bípedo' son accidentes separables de $X$; 2) b) 'animal' y 'bípedo' son accidentes inseparables de $X$. De ser verdadera 1) a), entonces 'animal' y 'bípedo' podrán pertenecer o no a 'hombre', de modo que podría haber 'hombre no-animal' y 'hombre no-bípedo' lo cual

33 Ver Charlton, p. 62.

34 Horstschäfer, T. M. “Über Prinzipien”, Berlin-New York: Walter de Gruyter, 1998, pp. 110-3. 
es absurdo ${ }^{35}$. Si evaluamos 1) b), es decir, que 'animal' y 'bípedo' sean inseparables de 'hombre', entonces la definición de 'hombre' estará contenida en la de 'animal' y en la de 'bípedo'. Esto es absurdo puesto que la definición del todo no puede estar contenida en la definición de las partes. Si tomamos 2) a), entonces 'animal' y 'bípedo' serán separables de un sujeto $X$, de modo que la conjunción 'animal bípedo' también será separable de $X$. Pero de ser así, dado que 'animal bípedo' es equivalente a 'hombre', entonces 'hombre' será un accidente, lo que está en contradicción con la premisa de la reducción al absurdo: “si 'hombre’ es ő $\pi \varepsilon \rho$ öv [...]”' (Física I, 3, 186b15-16). La última posibilidad 2) b), Aristóteles no la aborda. Sin embargo, podemos reconstruir el argumento de la siguiente manera: si 'animal' y 'bípedo' fuesen predicados del sujeto $X$ como 'ñata' es predicado de 'nariz', la definición de $X$ estaría contenida en la de 'animal' y en la de 'bípedo'. Ello llevaría al absurdo de que 'animal' y 'bípedo' solo serían predicables de $X$ y no de otro sujeto como, e.g., 'hombre'. De ser así, no podríamos afirmar que un hombre sea animal ni que sea bípedo.

La reducción al absurdo ha mostrado, entonces, que la premisa de la que parte la refutación, a saber, “'animal' y 'bípedo' no son ő $\pi \varepsilon \rho$ ő $v \imath$ ”, debe ser falsa. De este modo, Aristóteles demuestra refutativamente que, del mismo modo en que 'hombre' puede ser analizado en 'animal' y 'bípedo', el ő $\pi \varepsilon \rho$ ő $v$ puede ser analizado en ciertos ő $\pi \varepsilon \rho$ ő $\tau \imath$; y ya que estos últimos poseen la misma naturaleza que el ö $\pi \varepsilon \rho$ ő $v$ del que son partes, pueden ser igualmente divididos en otras partes conceptuales, cada una de las cuales será un ö $\pi \varepsilon \rho$ ǒv $\tau$. Aristóteles ha demostrado que, incluso tomado en el sentido más fundamental en que pudiese ser entendido, el Ser parmenídeo es divisible, de modo que la unidad del Ser no puede ser absoluta, sino que se ha de admitir cierta divisibilidad.

En consecuencia, ya que Aristóteles ha formulado la premisa parmenídea 'el ser se dice de una manera' como proposición contradictoria con su principio ontológico 'el ser se dice de muchas maneras', la refutación de la tesis eleata no parece constituir solo una refutación de la ontología monista de los eleatas, sino también una demostración refutatoria de la verdad del $\pi \mathrm{o} \lambda \lambda \alpha \chi \tilde{\omega} \varsigma$. Esta demostración refutatoria es un ejemplo del uso 'fuerte' de la dialéctica, es decir, de su utilidad científica. Ella permite a Aristóteles demostrar dialécticamente uno de los principios de su filosofía natural, a saber, la hipótesis de que existe la pluralidad y, de este modo, contribuye a probar la posibilidad de fundar un estudio filosófico de la naturaleza. Como hemos mencionado antes, la tesis eleata de la inmovilidad deriva de su tesis monista, de modo que la refutación de

35 El absurdo de la conclusión es más evidente en el caso de 'hombre no-animal' que en el caso de 'hombre no-bípedo'. En efecto, podría haber hombres que no fuesen bípedos. Aristóteles podría haber escogido definir 'hombre' como 'animal racional', en cuyo caso habría contado con 'animal' y 'racional'. Así habría sido inmediatamente evidente que no puede haber 'hombre no-animal' ni 'hombre no-racional'. Sin embargo, luego le habría acarreado dificultades para ilustrar el absurdo de considerar 'animal' y 'racional' como atributos inseparables de 'hombre', pues si bien la definición de 'hombre' no está contenida en la definición de animal, sí está contenida en la de 'racional'. 
esta última conlleva la refutación de la inmovilidad. Si bien Aristóteles aborda recién en Física I, 7-8 la refutación de esta tesis, el argumento de I, 2-3 puede ser considerado una prueba suficiente de su falsedad y, con ello, de la verdad de la segunda hipótesis fundamental de la física de Aristóteles: hay movimiento.

\section{Conclusiones y consideraciones finales}

El propósito de este trabajo ha sido mostrar que la refutación al monismo eleata, en Física I, 2-3, se justifica solo desde el punto de vista de las exigencias del método empleado por Aristóteles en su investigación sobre los principios de los entes naturales, es decir, desde las exigencias del método dialéctico 'fuerte'.

Física I es un tratado dialéctico que busca identificar los primeros principios de los entes naturales, pero que también busca establecer la posibilidad de llevar esto a cabo. Esto implica, en primer lugar, refutar las opiniones de otros filósofos que hayan negado esta posibilidad. En segundo lugar, implica probar la verdad de la hipótesis fundamental de la ciencia de la naturaleza, a saber: existencia de la pluralidad y el movimiento. Es en el marco de esta prueba donde la refutación al eleatismo se justifica en ambos sentidos, pues la tesis eleata constituye la tesis que niega la posibilidad misma de fundar una ciencia del ente natural, en la medida en que niega la hipótesis fundamental de la ciencia física aristotélica. De este modo, la refutación de la tesis eleata conlleva la anulación de la principal objeción filosófica contra el proyecto aristotélico y, además, constituye una prueba de la verdad de la hipótesis fundamental.

La refutación al eleatismo es una argumentación dialéctica, pues se basa en los tres procedimientos lógicos propios del método dialéctico de investigación científica: el análisis semántico, la diaporética y la reducción a contradicción. Analizando el sentido en que los eleatas entendieron el őv y el ह̌v , desarrollando las consecuencias de las premisas eleatas, y mostrando las contradicciones internas de su ontología y henología, Aristóteles demuestra refutativamente la falsedad de la tesis 'el ser se dice de una única manera'. Y ya que la tesis eleata es formulada como proposición que contradice directamente el principio ontológico 'el ser se dice de muchas maneras', la refutación de aquella implica la demostración refutatoria del $\pi \mathrm{o} \lambda \lambda \alpha \chi \tilde{\omega} \varsigma$.

Ahora bien, las argumentaciones de Aristóteles suponen siempre la verdad del $\pi \mathrm{0} \lambda \lambda \alpha \chi \tilde{\omega} \varsigma$, incluso cuando pretende argumentar únicamente desde la verdad hipotética de la tesis eleática. Dicho de otro modo, partiendo de la admisión hipotética de la verdad del $\mu$ ov $\alpha \chi \tilde{\omega} \varsigma$, Aristóteles intenta construir una reducción al absurdo que refute la tesis monista de los eleatas. Durante esta refutación, la verdad del $\pi \mathrm{o} \lambda \lambda \alpha \chi \tilde{\omega} \varsigma$ debe quedar en suspenso, pues, de lo contrario, se estaría negando la premisa de la reducción al

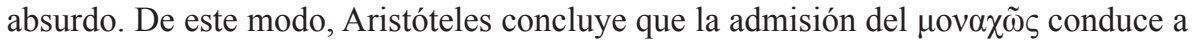
conclusiones absurdas, revelándose de este modo la verdad del $\pi \mathrm{o} \lambda \lambda \alpha \chi \tilde{\omega} \varsigma$. Ahora bien, el $\pi \mathrm{o} \lambda \lambda \alpha \chi \tilde{\omega} \varsigma$ es un principio tan fundamental, que su verdad nunca puede ser puesta entre paréntesis totalmente. Su admisión queda implícita en todo uso del lenguaje, en la medida en que se establezca alguna diferencia mínima entre dos cosas que son. En 
efecto, dejar absolutamente entre paréntesis el $\pi \mathrm{o} \lambda \lambda \alpha \chi \tilde{\omega} \varsigma$ supondría la imposibilidad de comenzar una reducción al absurdo como la que construye Aristóteles, ya que ella solo puede comenzar una vez establecida una premisa, en la que ya está implícita una diferenciación entre sujeto y predicado y, con ello, ya está implícita la admisión del $\pi \mathrm{o} \lambda \lambda \alpha \chi \tilde{\omega} \varsigma$. En consecuencia, si se atribuye a Aristóteles la pretensión de demostrar refutativamente la verdad de aquello que está implícito en su argumentación, podría ser acusado de cometer la falacia petitio principii.

No obstante lo anterior, lejos de acusar a Aristóteles de cometer esta falacia, hemos querido reconocer su intento por desarrollar un método que pretende probar, al menos de manera aproximada, la verdad de uno de los principios más fundamentales del conocimiento.

\section{Referencias bibliográficas}

Aristóteles (1993), Física. Libros I y II. Trad. Marcelo D. Boeri. Buenos Aires: Editorial Biblos. Gredos.

(1998), Metafisica. Trad. Tomás Calvo Martínez. Madrid: Editorial (1982), Metafísica. Trad. Valentín García Yebra. Madrid: Editorial Gredos.

(1989), Posterior Analytics; Topica. Trad. Tredennick, H; Forster, E.S. Harvard University Press.

(1982), Tratados de lógica I. Trad. Miguel Candel Sanmartín, Madrid: Editorial Gredos.

(1988), Tratados de lógica II. Trad. Miguel Candel Sanmartín. Madrid: Editorial Gredos.

(2004), Topik. Trad. Rapp, Ch.; Wagner, T. Stuttgart: Reclam.

(1979), Aristotelis Topica et Sophistici Elenchi. Ross, W.D., ed. Oxford: Classical Texts.

(1936), Aristotle. Physics. Ross, W.D., ed. Oxford: Clarendon Press.

(1997), Aristotle. Topics. Books I and VIII. Trad. R. Smith. Oxford: Clarendon Press.

Barnes, J. (1975), “Aristotle's Theory of Demonstration”. En Barnes, J.; Schofield, M.; Sorabji, R., ed., Articles on Aristotle, Duckworth.

Berti, E. (1991), "Les méthodes d'argumentation et de démonstration dans la "Physique" (apories, phénomènes, principes)", en La Physique d'Aristote et les conditions d'une science de la nature. Paris: Librairie Philosophique J. Vrin; pp. 53-72.

(2008), Las razones de Aristóteles. Trad. Horacio A. Gianneschi y Maximiliano Monteverdi. Buenos Aires: Oinos. 
Bolton, R. (2003), “Aristotle's Method in Natural Science: Physics I", en L. Judson, ed., Aristotle's Physics. A Collection of Essays. Oxford: Clarendon Press, pp. $1-29$.

Cassini, A. (1988), "El fundacionismo de la epistemología aristotélica". Crítica, Revista Hispanoamericana de Filosofía 20: 67-95.

Charlton, W. (1970), Aristotle's Physics I, II. Oxford: Clarendon Press.

Horstschäfer, T. M. (1998), Über Prinzipien. Berlin-New York: Walter de Gruyter.

Mansion, S. (1953), “Aristote, critique des Eléates”. Revue Philosophique de Louvain 51: $165-186$.

Platón (1988), Parménides. Trad. M. Isabel Santa Cruz. Madrid: Editorial Gredos. (1965), Parménide. Trad. Auguste Diès. Paris: Les Belles Lettres.

Sexto Empírico (1993), Esbozos Pirrónicos. Trad. Antonio Gallego Cao, Teresa Muñoz Diego. Madrid: Editorial Gredos.

Siggen, M. (2006), La Méthode Expérimentale selon Aristote. Les Presses Universitaires.

Wieland, W. (1992), Die aristotelische Physik. Göttingen: Vandenhoeck \& Ruprecht. 